\title{
LIVER
}

\section{Hepatitis $C$ virus replicates in peripheral blood mononuclear cells of patients with occult hepatitis $C$ virus infection}

\author{
I Castillo, E Rodríguez-Iñigo, J Bartolomé, S de Lucas, N Ortíz-Movilla, J M López-Alcorocho, \\ M Pardo, V Carreño
}

See end of article for authors' affiliations

Correspondence to

Dr V Carreño, Fundación para el Estudio de las Hepatitis Virales, Guzmán el Bueno, 72, 28015 Madrid, Spain; fehvhpa@ fehv.org

Revised version received 21 December 2004 Accepted for publication 14 January 2005
Background: Occult hepatitis $\mathrm{C}$ virus (HCV) infection is characterised by the presence of HCV-RNA in the liver in the absence of anti-HCV, and serum viral RNA. Up to $70 \%$ of these patients also have HCV-RNA in peripheral blood mononuclear cells (PBMC) but it is not known if HCV is replicating in these cells.

Aim: We studied possible HCV replication in PBMC of 18 patients with an occult HCV infection who were selected on the basis of HCV-RNA positivity in PBMC.

Methods: Detection of HCV-RNA positive and negative strands in PBMC was done by strand specific reverse transcriptase-polymerase chain reaction (RT-PCR) and by in situ hybridisation.

Results: The presence of HCV-RNA positive strand in PBMC was confirmed in all patients by strand specific RT-PCR and by in situ hybridisation. Mean percentage of PBMC which had the HCV-RNA positive strand was 3.3\% (95\% confidence interval (CI) 2.1-4.4) The HCV-RNA negative strand was found in the PBMC of $11 / 18(61 \%)$ patients by strand specific RT-PCR and confirmed by in situ hybridisation, and the percentage of PBMC harbouring the HCV-RNA negative strand was $3.1 \%(95 \% \mathrm{Cl} 0.8-5.5)$. There was a significant correlation $(p=0.001, r=0.84)$ between the percentage of PBMC with the HCV-RNA positive strand and that of PBMC with the HCV-RNA negative strand.

Conclusion: HCV replicates in the PBMC of patients with occult HCV infection and thus, although these patients do not have serum HCV-RNA, they could be potentially infectious.
$\mathrm{H}$ epatitis $\mathrm{C}$ virus (HCV) is a single stranded positive RNA virus belonging to the Flaviviridae family. ${ }^{1}$ Although the mechanism of HCV replication is not fully understood, it is assumed that virus replication involves the synthesis of a negative strand RNA molecule that acts as a template for production of positive strand or genomic HCV-RNA. ${ }^{2}$ Thus detection of the HCV-RNA negative strand is indicative of viral replication. The liver is the main site of virus replication but it can also replicate at extrahepatic sites such as peripheral blood mononuclear cells (PBMC). ${ }^{3-6}$ Regarding this infection of PBMC, it has been shown that HCV can propagate in lymphoid cell cultures and that the virus derived is infectious. ${ }^{78}$ In addition, it has been proposed that PBMC could be the source of recurrent HCV infection after liver transplantation. ${ }^{910}$

We recently described the existence of occult HCV infections, defined by the presence of HCV-RNA in the liver in the absence of anti-HCV and serum HCV-RNA. ${ }^{11}$ In addition, up to $70 \%$ of these patients also had HCV-RNA in their PBMC. ${ }^{11}$ As these patients do not have detectable circulating viral RNA, an important question with regard to transmission of occult HCV infection is whether HCV replicates in PBMC. Thus in the present work we investigated HCV replication in PBMC of patients with occult HCV infection by detection of HCV-RNA positive and negative strands using a strand specific reverse transcriptase-polymerase chain reaction (RT-PCR) and in situ hybridisation.

\section{PATIENTS AND METHODS}

In a previous report, ${ }^{11}$ we described a group of patients with abnormal liver function tests of unknown aetiology who had an occult HCV infection (HCV-RNA in the liver, as detected by RT-PCR and in situ hybridisation but anti-HCV and serum HCV-RNA negative). Among them, we selected for the present work those who: (i) were also HCV-RNA positive in the PBMC sample obtained on the same day of the liver biopsy and (ii) had available aliquots of that PBMC sample stored in liquid nitrogen. A total of 18 patients fulfilled the inclusion criteria and were included in this study, after giving written informed consent to participate. The characteristics of these 18 patients are shown in table 1 . None reported risk factors (blood transfusions, drug abuse, sexual behaviour, tattoos, etc) for HCV infection. Four of these 18 patients had abnormal levels of aspartate aminotransferase (AST), alanine aminotransferase (ALT), and gamma-glutamyl transpeptidase (GGTP) simultaneously; five had abnormal values of two liver enzymes (ALT and GGTP four patients; ALT and AST one patient), and the remaining nine patients had abnormal levels of only one liver enzyme (GGTP six patients and ALT three patients). PBMC samples from six healthy volunteers with normal liver function tests, who were repeatedly HCV-RNA negative in PBMC, were used as negative controls.

Total RNA was isolated from PBMC using the SV Total RNA Isolation System (Promega Corp., Madison, Wisconsin, USA) and, after precipitation, the RNA pellet was dissolved in diethyl-pyrocarbonate treated water. The amount of total RNA was determined by spectrophotometry and $0.5 \mu \mathrm{g}$ of RNA were used for detection of HCV-RNA of both polarities.

\section{Synthetic HCV-RNA}

Synthetic HCV-RNA positive and negative strands were generated by in vitro transcription of the recombinant plasmid $\mathrm{pC}^{\prime} \mathrm{NCR}$, which contains the complete $5^{\prime}$ noncoding region of the HCV genome. After plasmid linearisation, the RNA positive and negative strands were synthesised

Abbreviations: FISH, fluorescent in situ hybridisation; HCV, hepatitis $\mathrm{C}$ virus; AST, aspartate aminotransferase; ALT, alanine aminotransferase; GGTP, gamma-glutamyl transpeptidase; PBMC, peripheral blood mononuclear cells; RT-PCR, reverse transcriptase-polymerase chain reaction 
Table 1 Characteristics of the patients with occult hepatitis $C$ virus (HCV) infection included in the study

\begin{tabular}{ll}
\hline No of patients & 18 \\
Sex (M/F) & $13 / 5$ \\
Age (y) & $45.7(41.0-50.3)$ \\
BMI (kg/m $\mathrm{m}^{2}$ ) & $25.3(24.2-26.5)$ \\
Estimated duration of abnormal liver & $35.5(14.3-56.7)$ \\
function tests (months) & \\
AST (IU/I) & $36.7(23.1-50.3)$ \\
ALT (IU/I) & $54.2(30.7-77.7)$ \\
GGTP (IU/I) & $116.5(67.3-165.7)$ \\
HCV-RNA positive strand in hepatocytes (\%) & $3.7(2.0-5.5)$ \\
HCV-RNA negative strand in hepatocytes (\%) & $2.2(1.2-3.3)$ \\
\hline
\end{tabular}

Values are mean $(95 \%$ confidence interval of the mean) or number. BMI, body mass index; AST, aspartate aminotransferase; ALT, alanine aminotransferase; GGTP, gamma-glutamyl transpeptidase.

by transcription from the SP6 RNA polymerase promoter or the T7 RNA polymerase promoter, respectively. The plasmid template was removed by two rounds of digestion for 30 minutes at $37^{\circ} \mathrm{C}$ with $1 \mathrm{U} / \mathrm{\mu g}$ DNA of DNase I (Promega), followed by phenol-chloroform extraction. Concentrations of both HCV-RNA strand transcripts were determined spectrophotometrically and the absence of residual DNA was assayed by PCR without the RT step. Tenfold serial dilutions of the RNA templates were made in RNA extracted from a HCV negative cell line (HepG2) such that all dilutions had a final concentration of $0.5 \mu \mathrm{g}$ of total RNA.

\section{Strand specific RT-PCR}

Specific detection of HCV-RNA positive and negative strands was done by performing the corresponding DNA synthesis at high temperature using the thermostable enzyme Tth. Briefly, cDNA synthesis was carried out in $20 \mu \mathrm{l}$ of reaction buffer consisting of $50 \mathrm{pM}$ of the corresponding primer from the $5^{\prime} \mathrm{NC}$ region of the HCV genome, $1 \times$ RT buffer (Applied Biosystems, Foster City, California, USA), $1 \mathrm{mM} \mathrm{MnCl}_{2}$, $200 \mu \mathrm{M}$ of each deoxynucleoside triphosphate, and $5 \mathrm{U}$ of rTth (Applied Biosystems). After 20 minutes at $65^{\circ} \mathrm{C}, \mathrm{Mn}^{2+}$ was chelated with $8 \mu \mathrm{l}$ of the $10 \times$ chelating buffer (Applied Biosystems). Thereafter, $50 \mathrm{pM}$ of the opposite primer was added, the volume adjusted to $100 \mu \mathrm{l}$, and the $\mathrm{MgCl}_{2}$ concentration adjusted to $2.2 \mathrm{mM}$. Amplification was performed as follows: initial denaturing for two minutes at $94^{\circ} \mathrm{C}$ and 30 cycles of $94^{\circ} \mathrm{C}$ for 30 seconds, $55^{\circ} \mathrm{C}$ for 30 seconds, and $72^{\circ} \mathrm{C}$ for one minute followed by a final extension at $72^{\circ} \mathrm{C}$ for seven minutes. For detection of the HCV-RNA positive strand, the cDNA reverse primer was 5'CAT GGT GCA CGG TCT ACG AGA CC 3' and the forward primer was 5'GGC GAC ACT CCA CCA TGA ATC AC $3^{\prime}$. The same primers were used in reverse order for detection of the HCV-RNA negative strand.

A sample of the RT-PCR reaction ( $10 \mu \mathrm{l})$ was added to the second nested PCR and amplified for another 30 cycles, using as inner forward primer, 5'CTG TGA GGA ACT ACT GTC TT $3^{\prime}$, and as the reverse inner primer, 5'CTC GCA AGC ACC CTA TCA GG 3'. PCR products (266 bp) were visualised by $1.4 \%$ agarose gel electrophoresis.

During extraction, RT, and amplification steps, the guidelines of Kwok and Higuchi were carefully followed to prevent PCR contamination. ${ }^{12}$

\section{Detection of HCV-RNA positive and negative strands in PBMC by fluorescent in situ hybridisation}

The HCV-RNA positive strand was detected using a cRNA probe of negative polarity obtained by in vitro transcription of the pC5'NCR in the presence of digoxigenin-11-UTP (Roche Molecular Biochemicals, Indianapolis, Indiana, USA).
Detection of the HCV-RNA negative strand was performed with a complementary digoxigenin labelled cRNA probe spanning 390 nucleotides of the HCV core coding region, obtained by in vitro transcription of the pCcore plasmid.

For fluorescent in situ hybridisation (FISH), PBMC were resuspended in $\mathrm{KCl}(0.075 \mathrm{M})$ for 10 minutes at $37^{\circ} \mathrm{C}$, pelleted at $500 \mathrm{~g}$ for five minutes, and resuspended in $15 \mu \mathrm{l}$ of phosphate buffered saline. Resuspended cells were mixed with $35 \mu \mathrm{l}$ of low melting point agarose maintained at $37^{\circ} \mathrm{C}$, deposited onto a glass slide (that was previously precoated with $0.65 \%$ standard agarose and dried at $80^{\circ} \mathrm{C}$ ), covered with a cover slip, and allowed to solidify at $4^{\circ} \mathrm{C}$. Finally, coverslips were gently removed by immersing the slides in phosphate buffered saline at room temperature. Hybridisation with both HCV-RNA riboprobes was carried out at $42^{\circ} \mathrm{C}$ for 16 hours in a humid chamber. After hybridisation, samples were washed at $42^{\circ} \mathrm{C}$ in $2 \times \mathrm{SSC}, 0.5 \times \mathrm{SSC}$, and $0.1 \times \mathrm{SSC}$ ( 15 minutes each) $(20 \times$ SSC: $3 \mathrm{M} \mathrm{NaCl}, 3 \mathrm{mM}$ trisodium citrate). Digoxigenin labelled hybrids were detected with fluorescein isothiocyanate conjugate (Roche Molecular Biochemicals). Signal intensity was amplified using the Fluorescent Antibody Enhancer set for DIG detection kit (Roche Molecular Biochemicals). Cells were counterstained with 4-6 diamidino-2-phenylindole. Fluorescent signals were observed using a Nikon Eclipse E-400 light-microscope (Nikon Co., Tokyo, Japan) and the images were captured with a high resolution monochrome CCD camera (DIC-N; Wared Precision Instruments, Cambridge, UK) At least 2000 cells per sample were counted to obtain the percentage of positive cells.

\section{Statistical analysis}

Data analysis was performed using SSPS version 9.0 for windows (Chicago, Illinois, USA). Normality and homocedasticity of continuous variables were tested by the Komolgorov-Smirnov and Levene's tests, respectively. The mean was compared with the Student's $t$ (variables with normal distribution) or with the Mann-Whitney $U$ test (variables with non-normal distribution). Categorical variables were compared using the $\chi^{2}$ or Fisher's exact tests. Pearson (variables with Gaussian distribution) or Spearman (non-normally distributed variables) correlation coefficients were determined to study the existence of correlation between the variables. All tests performed were two sided and statistical significance was considered at a p value $<0.05$.

\section{RESULTS}

To assess the sensitivity and specificity of our strand specific RT-PCR assay, serial dilutions of synthetic HCV-RNA positive and negative strands were used as RNA templates. The analysis showed that the positive strand assay detected the HCV-RNA positive strand at $0.1 \mathrm{fg}$ and the HCV-RNA negative strand at $10 \mathrm{pg}$ (fig $1 \mathrm{~A}$ ). Thus, discrimination was 100000 -fold. Similarly, the negative stand assay detected the HCV-RNA negative strand at $1 \mathrm{fg}$ while the RNA positive strand was detected at $10 \mathrm{pg}$ (fig $1 \mathrm{~B}$ ), resulting in a $10000-$ fold discrimination.

The results obtained by strand specific RT-PCR assay as well as by the FISH technique confirmed that all 18 patients with occult HCV infection had the HCV-RNA positive strand in their PBMC. In contrast, PBMC from six healthy donors were negative. Mean percentage of PBMC showing fluorescent signals for the HCV-RNA positive strand was 3.3\% (95\% confidence interval (CI) 2.1-4.4)

When studying the presence of the RNA negative strand in PBMC, we found that $11 / 18(61 \%)$ patients were positive by the strand specific RT-PCR. The existence of the HCV-RNA negative strand was confirmed by FISH in these 11 cases while no hybridisation signals were seen in the PBMC of the 

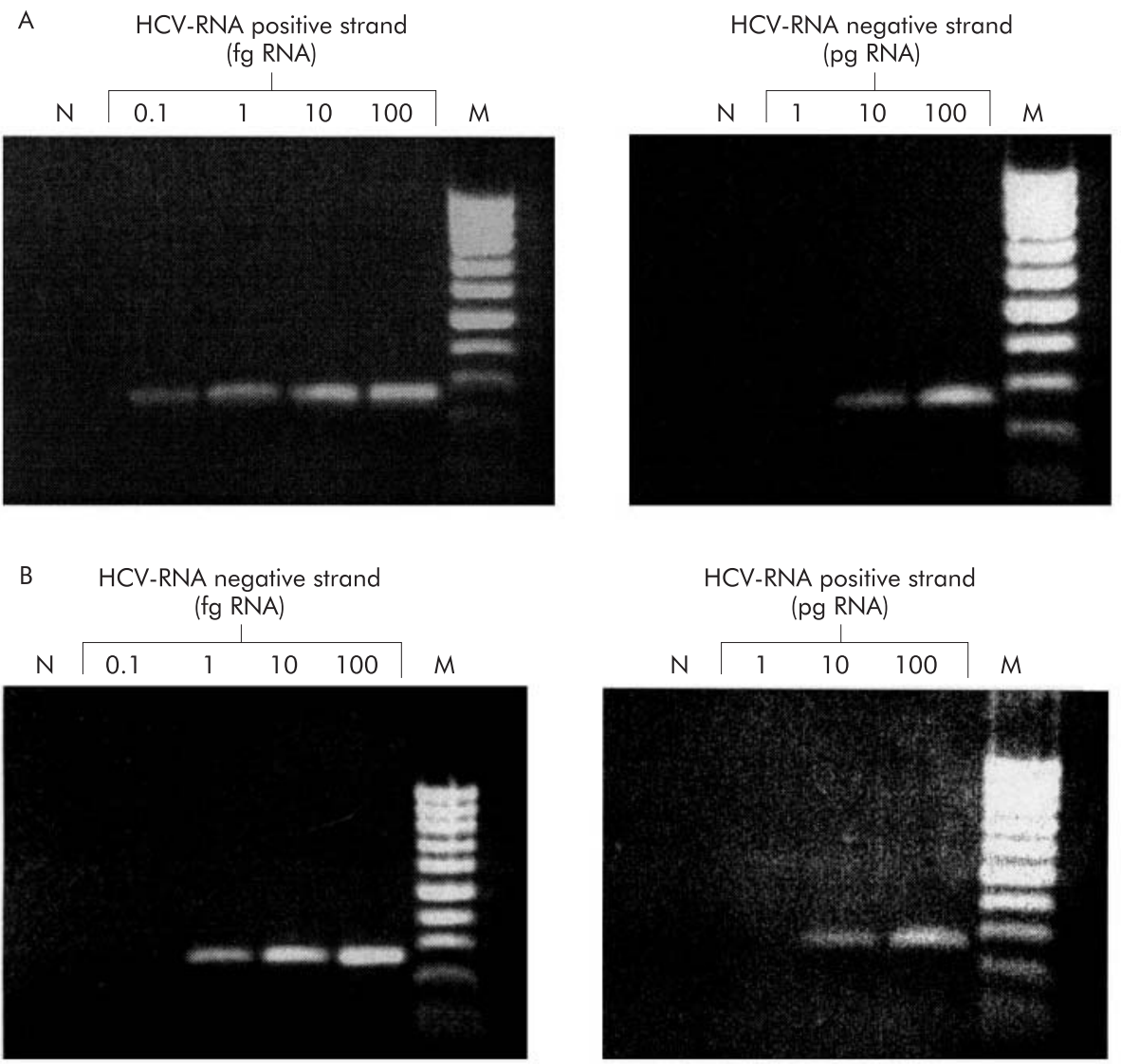

Figure 1 Specificity and sensitivity analysis of the strand specific reverse transcriptase-polymerase chain reaction using synthetic hepatitis $C$ virus (HCV)-RNA. (A) Positive strand RNA assay of HCV-RNA positive strand (0.1-100 fg) and HCV-RNA negative strand (1-100 pg). Lane N, negative control; lane M, 100 bp DNA ladder. (B) Negative strand RNA assay of HCV-RNA negative strand (0.1-100 fg) and HCV-RNA positive strand (1-100 pg). Lane N, negative control; lane M, 100 bp DNA ladder.

remaining seven patients. PBMC samples from the healthy donors were negative for the RNA negative strand by both techniques. In 11 patients with occult HCV infection, mean percentage of PBMC with a positive hybridisation signal for the HCV-RNA negative strand was 3.1\% (95\% CI 0.8-5.5).

When patients were divided according to the status of HCV replication in their PBMC, we found that the mean percentage of PBMC cells harbouring the HCV-RNA positive strand was significantly higher $(p=0.009)$ in those patients with HCV replication in PBMC (mean 4.2\% (95\% CI 2.5-5.9)) than in those without HCV replication (mean $1.8 \%$ (95\% CI 1.3-2.2)). No other differences were found between the groups of patients (table 2).

In contrast, there was a significant correlation $(\mathrm{p}=0.001$, $r=0.84$ ) between the percentage of PBMC with the HCVRNA positive strand and that of PBMC with the HCV-RNA

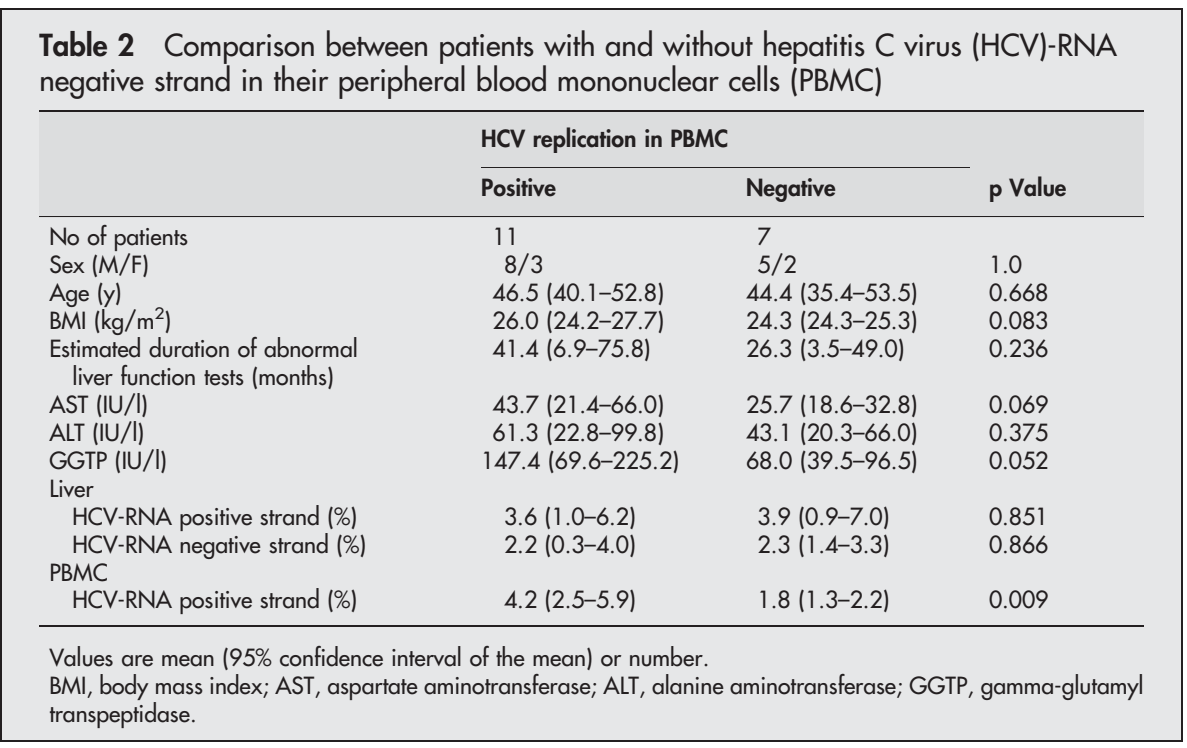




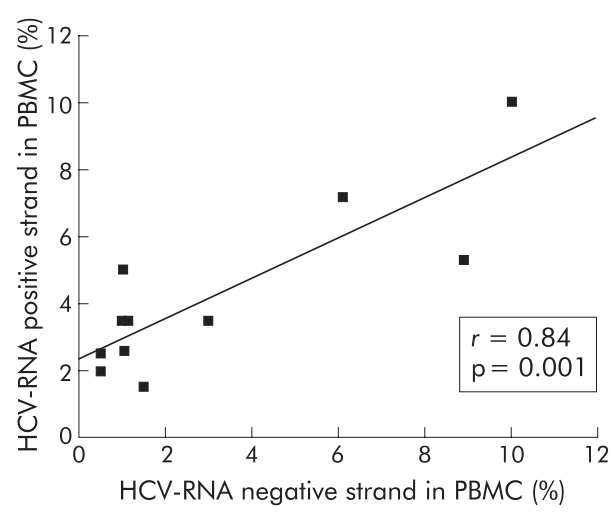

Figure 2 Correlation between percentages of peripheral blood mononuclear cells (PBMC) showing hybridisation signals for hepatitis $C$ virus (HCV)-RNA positive and negative strands.

negative strand (fig 2). However, no correlations were seen between the percentages of HCV-RNA positive or negative strands in PBMC and in hepatocytes. In addition, the existence of HCV replication in PBMC was not related to the presence of HCV replication in the liver (data not shown).

\section{DISCUSSION}

In the present work, we investigated if HCV replicates in the PBMC of 18 patients with occult HCV infection (who had HCV-RNA in their PBMC) by specific detection of viral RNA positive and negative strands using two techniques: a highly specific RT-PCR assay and FISH. The specificity of our RTPCR assay was assessed with serial dilutions of synthetic HCV-RNA positive and negative strands, showing $10000-$ 100000 -fold differentials between detection of the correct and incorrect strands of RNA. These differentials confirm the specificity of HCV-RNA negative strand detection in PBMC as in vivo the HCV-RNA positive-negative strand ratio is 10-100-fold. ${ }^{4}{ }^{13}$

Detection of the HCV-RNA positive strand by RT-PCR and FISH confirmed the existence of HCV infection in the PBMC of the 18 patients with occult HCV infection included in the study. Analysis of the HCV-RNA negative strand showed that HCV was replicating in the PBMC of the majority (61\%) of patients with occult HCV infection, with concordant results between the two different techniques. This percentage of HCV replication in PBMC of patients with occult HCV infection is similar to that reported in PBMC of patients with chronic hepatitis C. ${ }^{356}$

The existence of HCV infection and replication in PBMC in the absence of detectable serum HCV-RNA has also been described in anti-HCV positive patients years after spontaneous or antiviral therapy induced clearance of serum viral RNA and normalisation of transaminases. ${ }^{14}{ }^{15}$ As there is no detectable circulating viral RNA, it could be assumed that HCV infection and replication in PBMC may be related to the status of intrahepatic HCV-RNA. However, in these patients with occult HCV infection, we did not find any correlation between the percentage of hepatocytes showing the HCVRNA positive or negative strand and these percentages in PBMC. Moreover, the existence of HCV replication in PBMC was not related to the presence of HCV replication in the liver. Whether this lack of association reflects infection with
HCV variants of different tropisms or with different replication capacities is currently under investigation.

In contrast, the percentage of PBMC harbouring the HCVRNA positive and negative strands were highly correlated with each other $(r=0.84 ; \mathrm{p}=0.001)$ and the mean percentage of infected PBMC was significantly higher $(p=0.009)$ in patients with HCV replication in PBMC than in those without HCV replication. Taking into account that if replication occurs the HCV-RNA positive-negative ratio is within 10-100fold ${ }^{4}{ }^{13}$ perhaps in these negative cases HCV replication is taking place but at such a low level that detection is beyond the sensitivity of the assay.

In conclusion, our findings indicate that HCV replicates in the PBMC of patients with occult HCV infection. As it has been shown that HCV can propagate in lymphoid cell cultures and that the virus derived is infectious, ${ }^{78}$ it should be determined whether these patients (although they do not have detectable circulating virus) are potentially infectious.

\section{ACKNOWLEDGEMENTS}

This work was partially supported by a grant from the Fundación Accenture, Madrid, Spain.

\section{Authors' affiliations}

I Castillo, E Rodríguez-Iñigo, J Bartolomé, S de Lucas, N Ortíz-Movilla, J M López-Alcorocho, M Pardo, V Carreño, Fundación para el Estudio de las Hepatitis Virales, Madrid, Spain

Conflict of interest: None declared.

\section{REFERENCES}

1 Miller RH, Purcell RH. Hepatitis C virus shares amine acid sequence similarity with pestiviruses and flaviviruses as well as members of two plant virus supergroups. Proc Natl Acad Sci U S A 1990;87:2057-61.

2 Clarke B. Molecular virology of hepatitis C virus. J Gen Virol 1997; 78:2397-410

3 Manzin A, Candela M, Paolucci S, et al. Presence of hepatitis C virus (HCV) genomic RNA and viral replicative intermediates in bone marrow and peripheral blood mononuclear cells from HCV-infected patients. Clin Diagn Lab Immunol 1994; 1:160-3.

4 Wang JT, Shen JC, Lin JT, et al. Detection of replicative from of hepatitis C virus RNA in peripheral blood mononuclear cells. J Infect Dis 1992:166:1167-9.

5 Saleh MG, Tibbs CJ, Koskinas J, et al. Hepatic and extrahepatic hepatitis C virus replication in relation to response to interferon therapy. Hepatology 1994;20:1399-404.

6 Chang $\Pi$, Young KC, Yang YJ, et al. Hepatitis C virus RNA in peripheral blood mononuclear cells: comparing acute and chronic hepatitis $C$ virus infection. Hepatology 1996;23:977-81.

7 Shimizu YK, Iwamata A, Hiijikata $M$, et al. Evidence for in vitro replication of hepatitis $C$ virus genome in a human T cell line. Proc Natl Acad Sci U S A 1992;89:5477-81.

8 Shimizu YK, Igarashi $\mathrm{H}$, Kiyahura $\mathrm{T}$, et al. Infection of a chimpanzee with hepatitis C virus grown in cell culture. J Gen Virol 1998;79:1383-6.

9 Feray C, Samuel D, Thiers V, et al. Reinfection of liver graft by hepatitis $C$ virus after liver transplantation. $J$ Clin Invest 1992;89:1361-5.

10 Laskus T, Radkowski M, Wilkinson J, et al. The origin of hepatitis C virus reinfecting transplanted livers: serum-derived versus peripheral blood mononuclear cell-derived virus. J Infect Dis 2002;185:417-21.

11 Castillo I, Pardo M, Bartolomé J, et al. Occult hepatitis C virus infection in patients in whom the etiology of persistently abnormal results of liver-function tests is unknown. J Infect Dis 2004;189:7-14.

12 Kwok S, Higuchi R. Avoiding false positive with PCR. Nature 1989;339:237-8

13 Radkowski M, Wang LF, Vargas HE, et al. Detection of hepatitis C virus replication in peripheral blood mononuclear cells after orthotopic liver transplantation. Transplantation 1998;66:664-6.

14 Maratori L, Giostra F, Cataleta M, et al. Testing for hepatitis $C$ virus sequences in peripheral blood mononuclear cells of patients with chronic hepatitis $C$ in the absence of serum hepatitis C virus RNA. Liver 1994;14:124-8.

15 Pham TNQ, MacParland SA, Mulrooney PM, et al. Hepatitis C virus persistence after spontaneous or treatment-induced resolution of hepatitis $C$. J Virol 2004;78:5867-74 